\title{
Die Rolle von Stressproteinen bei der Anpassung an Umweltbedingungen: Ökophysiologische, ökotoxikologische und evolutionsbiologische Implikationen
}

\author{
Heinz-R. Köhler
}

Eingegangen: 27. November 2008/Akzeptiert: 22. Februar 2009/Online veröffentlicht: 20. März 2009

(C) Springer-Verlag 2009

Zusammenfassung Einleitung Stressproteine sind ubiquitär verbreitet und durch eine Vielzahl von Stressoren induzierbar. Seit Beginn der 1990er Jahre wird ihre Bedeutung für ökologische und mikroevolutive Anpassungen untersucht.

Stressproteine: Funktion und Induktion Es existieren verschiedene Familien von Stressproteinen, wobei die meisten Daten für die Familien Hsp70, Hsp60 und Hsp90 vorliegen. Es handelt sich bei diesen Proteinen um cytoplasmatische Chaperone, die eine zentrale Rolle bei der korrekten Faltung von Polypeptiden, bei deren Transmembranpassage und bei der Stabilisation von Signaltransduktionsprozessen besitzen. Hsp70 wird durch erhöhte intrazelluläre Konzentrationen ungefalteter oder falsch gefalteter Proteine induziert und kann somit proteotoxische Wirkungen indizieren.

Modulation des Stressproteinlevels durch interne Faktoren Während bestimmter Entwicklungsstadien werden Stressproteinlevel durch interne Faktoren verändert. Dies wurde insbesondere für die frühe Individualentwicklung, die Formation von Ruhestadien und die Reproduktionsphase gezeigt.

Anpassung an äußere Stressoren unter Laborbedingungen In Laborversuchen zur Wirkung ökophysiologisch relevanter Stressoren wurde für eine Reihe von Taxa nachgewiesen, dass eine kurzzeitige physiologische Anpassung an erhöhte Temperaturen sowie diverse Umweltschadstoffe eine Erhöhung des Stressproteinlevels nach sich zieht. Bei extremer Stresseinwirkung bricht dieser Reaktionsmechanismus zusammen.

Herrn Prof. Dr. Dr. h. c. Volker Storch mit bestem Dank zum 65. Geburtstag gewidmet.

Herausgeber: Henner Hollert · Thomas Braunbeck

H.-R. Köhler $(\bowtie)$

Universität Tübingen, Physiologische Ökologie der Tiere,

Konrad-Adenauer-Str. 20, 72072 Tübingen, Deutschland

E-Mail: heinz-r.koehler@uni-tuebingen.de
Anpassung an Freilandbedingungen Auch im Freiland sind in die physiologische Adaptation an Temperaturschwankungen und Kontaminationen i.d. R. Induktionen von Stressproteinen involviert, die als Effektmarker proteotoxischer Wirkungen diagnostisch genutzt werden können.

Evolutionsbiologische Implikationen In langzeitbelasteten Gebieten werden jedoch vielfach Phänotypen mit niedrigen Stressproteinleveln evolutionär begünstigt. Es wird diskutiert, ob unterschiedliche Strategien bei der Stressantwort aufgrund der Rolle von Hsps bei der Kanalisation von Entwicklungsprozessen unterschiedliche Grade phänotypischer Variation auch unter natürlichen Verhältnissen bedingen.

Schlüsselwörter Biomarker · Canalization · Hsp · Mikroevolution · Ökotoxikologie · physiologische Anpassung · Proteotoxizität $\cdot$ Stress $\cdot$ Stressproteine $\cdot$ Toleranz

The role of stress proteins in the adaptation to the environment: implications for ecophysiology, ecotoxicology, and evolutionary biology

Abstract Introduction Stress proteins are ubiquitous and inducible by a vast number of stressors. Their relevance for ecological and microevolutionary adaptations has been investigated since the early 1990s.

Stress proteins: function and induction Stress proteins are categorized in different families of which most data have been recorded for Hsp70, Hsp60, and Hsp90. These cytoplasmatic proteins play a central role in protein folding, the trans-membrane passage of proteins, and the stabilization of signal transduction pathways. Hsp70 is induced by elevated concentrations of unfolded or malfolded intracellular proteins and hence indicates proteotoxicity. 
Modulation of the stress protein level by internal factors During certain developmental stages, stress protein levels are modified by internal factors. This has been particularly shown for the early ontogeny, for the formation of quiescent stages, and for the reproductive phase.

Adaptation to external stressors in the laboratory Laboratory studies on the effects of ecophysiologically relevant stressors have revealed short-term physiological adaptation to heat or environmental chemicals to involve an elevation of stress protein levels. Extreme stressor impact disrupts this responsive mechanism.

Adaptation to field situations Also in the field, physiological responses to temperature fluctuations or contamination usually involve the induction of stress proteins which can be used as a biomarker of proteotoxic effect.

Implications for evolutionary biology In long-term contaminated sites, however, phenotypes displaying low stress protein levels appear to be evolutionarily favored. Whether different stress response strategies may entail different degrees of phenotypic variation even under natural conditions - via Hsp action on developmental canalization - is a matter of hot debate.

Keywords Biomarkers · Canalization · Hsp · Microevolution Ecotoxicology $\cdot$ Physiological adaptation $\cdot$ Proteotoxicity $\cdot$ Stress $\cdot$ Stress proteins $\cdot$ Tolerance

\section{Einleitung}

Im Jahr 1962 entdeckte F. Ritossa, dass plötzliche Temperaturerhöhung bei Drosophila-Larven zu einer Änderung des Puffing-Musters führte, welches später als Ausdruck differentieller Genaktivität erkannt wurde. Die korrespondierenden Proteine wurden erst zwölf Jahre später von Tissières et al. (1974) beschrieben und entsprechend des ihrer Induktion zu Grunde liegenden Stressors, Temperaturerhöhung a als Hitzeschockproteine (Heat shock proteins, Hsp) bezeichnet. Schon bald zeigte sich jedoch, dass die Induktion dieser Proteine nicht allein auf Temperatureffekte, sondern vielmehr auf die Wirkung zahlreicher Stressoren unterschiedlichster Natur (z. B. auch Schwermetalle, Viren, Aminosäureanaloga etc.) zurückzuführen war, und so wird heute die Bezeichnung ,Stressproteine' für diese Gruppe von Proteinen bevorzugt, wobei jedoch das Kürzel Hsp blieb. Stressproteine sind eine Gruppe molekularer Chaperone, d.h. Proteine, die an der Aufrechterhaltung essentieller zellulärer Prozesse beteiligt sind. Sie sind dementsprechend phylogenetisch sehr alt, in manchen Hsp-Familien strukturell äußerst hoch konserviert und wurden bisher in allen darauf untersuchten Taxa der belebten Natur (Eubakterien, Archaebakterien, Protisten, Pflanzen, Pilzen und Tieren) nachgewiesen. Ihre molekularen und zellphysiologischen Funktionen wurden ausführlich in unterschiedlichsten Gebieten der Lebenswissenschaften untersucht und sind Gegenstand zahlreicher Review-Artikel (z.B. Nover 1984; Gething und Sambrook 1992; Parsell und Lindquist 1993; Feder und Hofmann 1999; Morimoto et al. 1999; Pockley 2003). Zu Beginn der 1990er-Jahre entstanden die ersten Arbeiten, die damit begannen, die Rolle dieser Proteine in der Stressökologie und Ökotoxikologie zu untersuchen (z. B. Bradley 1990; Köhler et al. 1992; Sanders 1993), und die somit die Basis für ihre Nutzung als Biomarker (suborganismische Reaktionen auf Umweltbelastungen) für Umwelteinflüsse legten (zusammengefasst in Kammenga et al. 2000; Sørensen et al. 2003). Seit dieser Zeit ist die Anzahl auch der ökologisch (und in neuerer Zeit auch evolutionsbiologisch) orientierten Publikationen zu diesem Thema kontinuierlich gestiegen, und diese können unmöglich in aller Ausführlichkeit in einem Kurz-Review wie dem vorliegenden Artikel dargestellt werden. Insofern orientiert sich der vorliegende Artikel vorzugsweise an Arbeiten der Arbeitsgruppe des Autors, die in den jeweiligen zeitlichen und inhaltlichen Kontext gestellt werden.

\section{Stressproteine: Funktion und Induktion}

Stressproteine werden entsprechend ihres Molekulargewichts verschiedenen Proteinfamilien zugeteilt. Neben den strukturell sehr konservierten Familien Hsp60, Hsp70 und Hsp90 (die Ziffern bezeichnen jeweils das ungefähre Molekulargewicht in $\mathrm{kDa}$ ) existieren eine eher heterogene Gruppe niedermolekularer Hsps mit einem Molekulargewicht unter $30 \mathrm{kDa}$ (low molecular weight (LMW) Hsps), Hsp40 und Hsp100 (auch high molecular weight (HMW) Hsps genannt) mit unterschiedlichen zellulären Funktionen. In der Ökologie und Evolutionsbiologie beschränkte sich die Forschung bisher jedoch weitgehend auf die Familien Hsp60, Hsp70 und Hsp90. Hsp70 ist hierbei die am besten untersuchte Familie, deren Induktionsmechanismus weitgehend verstanden ist (Abb. 1). Die Isoformen der Hsp70-Familie sind cytoplasmatische Proteine, die in der Regel nicht isoliert, sondern an ein weiteres Protein, Hsf (heat shock factor, wiederum in verschiedenen Isoformen) gebunden vorliegen. Mit Hsf konkurrieren allerdings auch ,ungefaltete' Polypeptidstränge unabhängig von ihrer Primärsequenz kompetitiv um die Bindungsstelle des Hsp70, so dass bei einer hohen intrazellulärer Konzentration ungefalteter oder falsch gefalteter Proteine vermehrt Hsp70 an derartige Strukturen bindet und in Konsequenz Hsf vermehrt freigesetzt wird. Dieses bindet wiederum in trimerisierter und phosphorylierter Form an hse (heat shock element)-Sequenzen der DNA und induziert die Transkription der $h s p 70$-Strukturgene. Nach erfolgter Translation steigt somit der intrazelluläre Level an Hsp70 so lange, bis der Induktor Hsf wieder quantitativ durch neu produziertes Hsp70 gebunden und die beschriebene Induk- 
tionskaskade wieder durchbrochen wird. Eine Erhöhung des Hsp70-Levels findet somit dann statt, wenn sowohl verstärkt neue (noch ungefaltete) Proteine synthetisiert werden oder wenn die Integrität intrazellulärer Proteine (durch partielle Denaturierung) beeinträchtigt wird. Letzterer Effekt wird als Proteotoxizität bezeichnet und ist vor allem bei der Diagnostik von Umweltstress von Bedeutung. Allerdings muss auch die Rolle von Stressproteinen im ,normalen` Zellmetabolismus bei der Indikation proteotoxisch wirkender Umweltbedingungen berücksichtigt werden. Basierend auf der Vielfalt möglicher Auslöser von Proteotoxizität ist somit die Induktion von Stressproteinen nicht dazu geeignet, auf die Natur des zu Grunde liegenden Stressors zu schließen. Stressproteine eignen sich jedoch insbesondere zur Beurteilung von (proteotoxischen) Effekten adverser Bedingungen, wobei über die kombinierte Wirkung mehrerer gleichzeitig wirkender Stressoren integriert wird (z. B. Köhler et al. 2001). Die Induktion von Stressproteinen ist demnach als biomarker of effect (und nicht biomarker of exposure) zu bezeichnen.

Die biochemische Funktion von Hsp70 wie Hsp60 besteht in einer ,Assistenz' bei der korrekten Faltung intrazellulärer Proteine, was die Bezeichnung, Chaperone' für beide Stressproteinfamilien zum Ausdruck bringen soll. Desweiteren ist Hsp70 an der Transmembranpassage von Polypeptiden beteiligt. Das ebenfalls phylogenetisch stark konservierte Hsp90 stabilisiert Signaltransduktionswege, so z.B. die Bindung (und somit auch Signaltransduktion) von Steroidhormonen an ihre Rezeptoren oder von planaren organischen Verbindungen an den Arylhydrocarbon-Rezeptor.

In der Literatur wird oftmals zwischen, stressinduzierbaren' Isoformen (z. B. Hsp70 sensu stricto) und nicht induzierbaren, stets auf gleichem Level verbleibenden Isoformen (z. B. Hsc70 $=70 \mathrm{kDa}$ heat shock cognate) unterschieden. Hierbei sollen nur die cognate-Gene Introns enthalten, da aufgrund der Anfälligkeit der Splicing-Maschinerie für disrupti-

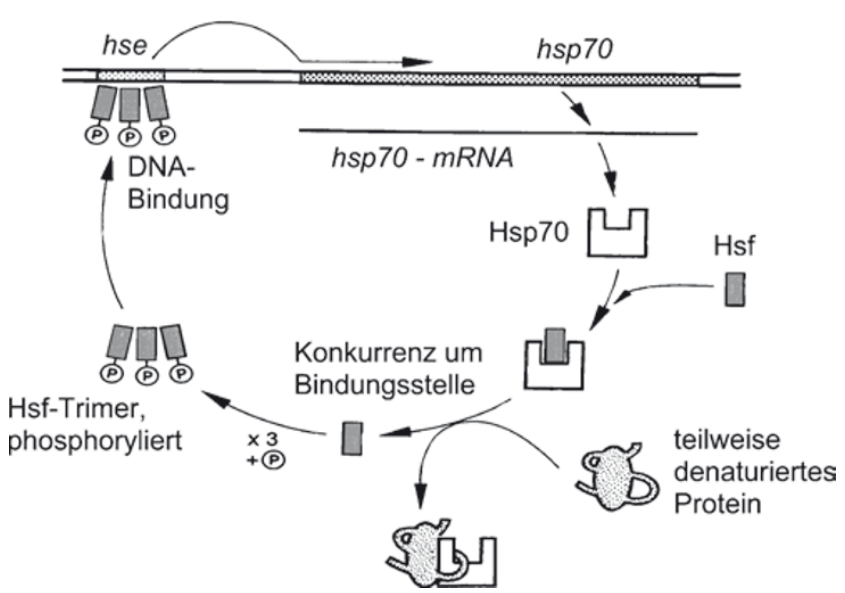

Abb. 1 Regulation der Induktion von Hsp70, vereinfacht, schematisch. Erklärung im Text ve Ereignisse und des durch Splicing erhöhten Zeitaufwands ein Selektionsdruck in Richtung Intronverlust bei stressinduzierbaren Stressgenen bestehen soll (Yost und Lindquist 1986, 1991). Allerdings ist die Unterscheidung zwischen ,induzierbaren' und cognate-Genprodukten vage und auch das Kriterium Besitz/Verlust von Introns wohl nicht universell anwendbar (Knigge 2003). Insofern konzentriert sich die ökophysiologische und ökotoxikologische Literatur nahezu ausschließlich auf Stressproteinfamilien und nicht auf einzelne Isoformen. Somit werden auch in diesem Artikel die Bezeichnungen Hsp70, Hsp90 und Hsp60 im Folgenden für die gesamten jeweiligen Stressproteinfamilien gebraucht.

\section{Modulation des Stressproteinlevels durch interne Faktoren}

Wie dargestellt, werden Stressproteine nicht nur durch proteotoxische Wirkungen, sondern auch in Lebensphasen erhöhter Translationsaktivität induziert. Die Kenntnis dieser internen Variabilität ist vor allem dann unerlässlich, wenn eine Änderung des Stressproteinlevels als diagnostisches Mittel (Biomarker) zur Indikation toxischer Wirkungen eingesetzt werden soll. Insbesondere unter diesen Umständen sollte der Einfluss der folgenden, durch Zitate exemplarisch belegten internen Faktoren berücksichtigt werden:

Frühe Ontogenese: In frühen Juvenilstadien besteht eine starke Notwendigkeit zur Neusynthese von Proteinen. So wiesen Larven des Zebrabärblings (Danio rerio) im Alter von 84 Stunden nach Fertilisation einen Hsp70-Level auf, der bis zu achtmal höher war als derjenige bei 12 Stunden alten Individuen (Yeh und Hsu 2002). Starke Erhöhungen der Level an Hsp70 und Hsp60 konnten auch in den quieszenten Stadien vor den jeweiligen Nymphenhäutungen bei der oribatiden Milbe Archegozetes longisetosus nachgewiesen werden (Köhler et al. 2005).

Reproduktionsphase: Der Flohkrebs Gammarus fossarum zeigte eine starke Abhängigkeit der Gehaltes an Hsp70 und Hsp90 vom reproduktiven Zyklus. So wiesen Individuen beiderlei Geschlechts direkt von der Eiablage/Insemination hohe Level an Hsp70 und niedrige Werte für Hsp90 auf; wenige Wochen später hatte sich die Situation umgekehrt (Schirling et al. 2004). In einer Freilandpopulation der Mützenschnecke Ancylus fluviatilis konnten über drei Jahre hinweg im Juli und November vor dem Auftreten neuer Juvenilgenerationen hohe Hsp70-Level in den Individuen der Parentalgeneration nachgewiesen werden (Adam, Köhler und Triebskorn, unpubliziert, Abb. 2).

Ruhestadien: Auch Stadien sehr geringer Stoffwechselaktivität können mitunter hohe Stressproteinlevel aufweisen. Gemmulae des Süßwasserschwamms Spongilla lacustris enthielten erstaunlich viel Hsp70-Protein und korrespondierende mRNA (Schill et al. 2006). Während 
Abb. 2 Größe und Hsp70Level abundanter Individuen einer Population der Mützenschnecke Ancylus fluviatilis aus einem Bach in Süddeutschland (Burtelbach, Württemberg). Die einer Generation zuzuordnenden Individuen sind in grober Annäherung durch Ellipsen markiert und durch Geraden miteinander verbunden. Die Hsp70-Level wiesen zwischen November 1997 und 1999 jeweils im Juli und November saisonale Maxima auf

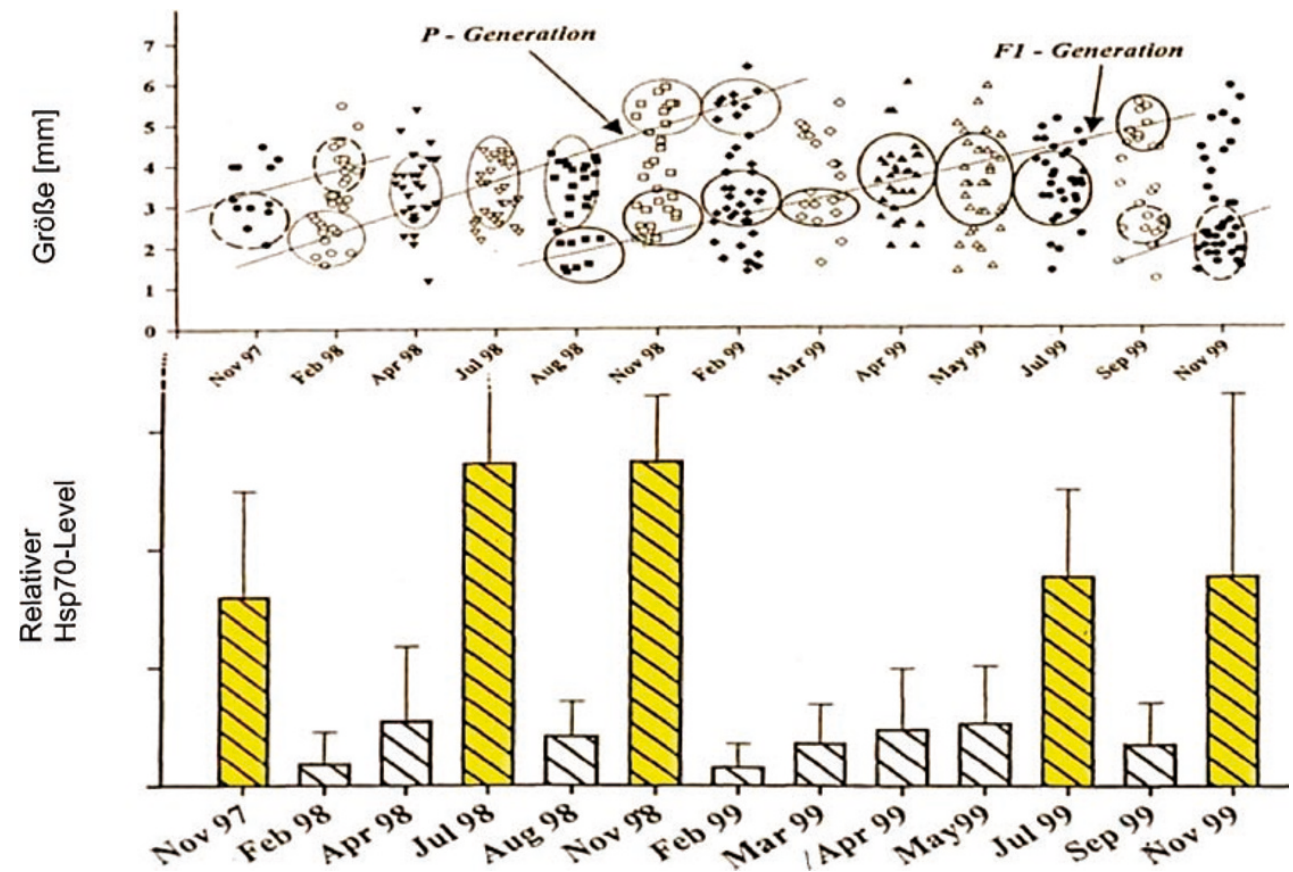

der Ausbildung cryptobiotischer Stadien des Tardigraden Milnesium tardigradum (die üblicherweise als stoffwechselfrei gelten) indizierten erhöhte mRNA-Level sogar die Induktion der Transkription einer Isoform von hsp70 (Schill et al. 2004). Auch in Diapause-II-Stadien des ,killifish 'Austrofundulus limnaeus konnten erhöhte Gehalte einer Hsp70Isoform nachgewiesen werden (Podrabsky und Somero 2007)

\section{Anpassung an äußere Stressoren unter Laborbedingungen}

Seit inzwischen mehr als dreißig Jahren ist bekannt, dass viele Stressoren unter Laborbedingungen eine molekulare Stressantwort, welche mit der Induktion von Stressproteinen einhergeht, induzieren können (Review-Artikel von Ashburner und Bonner 1979; Nover 1984). Seit dieser Zeit wurden über 100.000 Fachartikel (nach Web of Science) zu diesem Thema veröffentlicht. Die meisten dieser Artikel beschäftigen sich allerdings nicht mit ökologischen oder evolutionsbiologischen Fragestellungen. Nichtsdestotrotz existiert mittlerweile eine recht gute Datenbasis zur Induzierbarkeit von Stressproteinen durch umweltrelevante Faktoren, wobei größtenteils erhöhte Umgebungstemperaturen oder umweltrelevante Schadstoffe untersucht wurden. Unstrittig ist, dass eine temperaturinduzierte Induktion von Stressproteinen bei verschiedensten Taxa (Pflanzen, Insekten, Fischen und Säugern) zu einer erhöhten Hitzetoleranz führte (Ulmasov et al. 1993; Gehring und Wehner 1995; Dahlgaard et al. 1998; Basu et al. 2002; Sun et al. 2002). Hinsichtlich Umwelt- schadstoffe existieren bei einer Vielzahl von Organismen Laborstudien zur Wirkung von Schwermetallen (Köhler et al. 1992; Köhler et al. 1996; Zanger et al. 1996; Eckwert et al. 1997; Schill et al. 2003), aromatischen Organika (Köhler et al. 1999b; Weber und Janz 2001; Cruz-Rodriguez und Chu 2002; Weber et al. 2002; Zheng et al. 2008), Pestiziden (Köhler et al. 1999b; Nazir et al. 2003; Mukhopadhyay et al. 2006; Saradha et al. 2008; Eder et al. 2009; Scheil et al. im Druck) und, seit den letzten Jahren, auch zu Lösungsmitteln (Hallare et al. 2004; Hallare et al. 2006) und Pharmazeutika bzw. Kosmetika (Hallare et al. 2004; Haap et al. 2008; Scheil et al. 2008). Die Induktion von Stressproteinen stellt hierbei eine Reaktion des Organismus auf den proteotoxischen Effekt des jeweiligen Stressors dar, wobei diese Reaktion sehr differenziert und nicht, wie früher vermutet, über einen An/Aus-Mechanismus erfolgt (Sørensen und Loeschcke 2001; Kristensen et al. 2002). Allerdings kann eine Erhöhung des Hsp-Levels nicht in beliebiger Intensität erfolgen. Sehr starke Intensitäten von Stressoren überlasten die molekulare Stressantwort und führen in der Folge zu Erniedrigungen der Stressproteinlevel, was nicht notwendigerweise, jedoch oftmals auf pathologische Zellschäden zurückzuführen ist (Zanger und Köhler 1996; Eckwert et al. 1997; Werner und Hinton 1999; Köhler et al. 2001). Die Induktionskinetik von Stressproteinen in Abhängigkeit von der Stressintensität (z. B. Konzentration von Schadstoffen) folgt also einer Optimumkurve, was aufgrund theoretischer Überlegungen generell für biochemische Stressreaktionen zutreffen muss. Die Kenntnis einer derartigen Kinetik ist jedoch für die Interpretation von Befunden im Freiland, in welchem üblicherweise viele interagierende Faktoren auf die 
Stressreaktion Einfluss nehmen, unabdingbar. Exemplarisch wurden auch in Laborversuchen Kombinationen verschiedener Stressoren (Temperatur/Chemikalien/Pathogene bzw. Parasiten) auf ihre Wirkung auf das Hsp-System untersucht (Hallare et al. 2005b; Sures und Radszuweit 2007; Eder et al. 2007), was z.T. half, Freilandbefunde einzuordnen (Köhler und Eckwert 1997). Eine Validierung der Bedeutung erhöhter Stressproteinlevel oder erhöhter Transkriptionsraten von Stressgenen für populationsbiologisch relevante Parameter, wie z. B. Mortalität oder Reproduktionsrate, fand bisher nur in Einzelfällen statt (Köhler et al. 1998).

\section{Anpassung an Freilandbedingungen}

Eine Simulation von Freilandbedingungen im Laborversuch ist außerordentlich schwierig. Selbst wenn Chemikalien auf der Basis von ausführlichen chemischen Analysen im Laborversuch eindosiert oder über Monate etablierte Mesokosmen (Fließrinnen) eingesetzt werden, entspricht die Stressantwort der exponierten Organismen nur ansatzweise den Verhältnissen im Freiland (Köhler et al. 2001; Schirling et al. 2006; Werner et al. 2006). Lediglich besonders naturnahe Mesokosmen (z.B. Bypass-Systeme für Fließgewässer, die von natürlichem Bachwasser durchströmt werden und somit die natürliche Variabilität der Expositionsbedingungen vergleichsweise gut abbilden) gewährleisteten gute Übereinstimmungen mit Freilandbedingungen (Köhler et al. 2001). Wie nachgewiesen werden konnte, existieren saisonal (Fader et al. 1994; Hofmann und Somero 1995; Minier et al. 2000; Köhler et al. 2001), über wenige Tage hinweg (Nguyen et al. 1994; Ferguson et al. 1998) oder in manchen Habitaten (z. B. durch Gezeiten beeinflusste Meeresküsten) sogar im tageszeitlichen Rhythmus (Schill et al. 2002; Schill et al. 2008) z. T. erhebliche Variationen im Level von Stressproteinen, die aus der Notwendigkeit physiologischer Anpassung an ökologische Bedingungen resultieren. Nichtsdestotrotz ist es unter vergleichsweise, stabilen ' Bedingungen (Beprobungen in einem Zeitraum von nur wenigen Wochen, Untersuchungen in tropischen/subtropischen Ökosystemen) möglich, auch unter Freilandbedingungen verlässliche Aussagen zu proteotoxischen Potentialen in der Umwelt zu erzielen. So konnten Werner und Hinton (2000) und Werner (2004) bei der Muschel Potamocorbula amurensis einen Einfluss der Salinität zeigen. Hallare et al. (2005a) wiesen nach, dass Wasserproben aus Laguna Lake in der Nähe von Manila, Philippinen, Hsp70 bei experimentell exponierten Zebrabärblingen induzieren. Abundante Fische (Oreochromis sp., Clarias sp.) aus Lake Mariut, Ägypten, zeigten in kontaminierten Regionen im Vergleich zu Individuen einer Kontrollstelle erhöhte Hsp70-Level in Kiemen und Lebern (Abdel-Latif, Köhler, Schill und Triebskorn, unveröffentlicht). Trotz noch größerer Heterogenität von
Fließgewässern existieren auch hier einige Beispiele erfolgreicher Bioindikation bei passivem Biomonitoring. In einer fünfjährigen Studie an zwei kleinen unterschiedlich belasteten Fließgewässern im süddeutschen Raum, Körsch und Krähenbach (Triebskorn et al. 2001), wiesen Bachforellen (Salmo truttaf. fario) und Bachschmerlen (Barbatula barbatula) deutlich unterschiedliche jahreszeitliche Muster hepatischer Hsp70-Level im Vergleich untereinander und zur Kontrolle auf (Köhler et al. 2001). Auch durch Kurzzeitstudien an Fließgewässern gelang es über die Bestimmung des Hsp70-Levels, Stellen erhöhter Proteotoxizität zu detektieren, so in einer Studie an Nase (Chondrostoma nasus) und Döbel (Leuciscus cephalus) im Unterlauf der Mureş, Rumänien (Köhler et al. 2007) und einer Untersuchung am Lachs (Salmo salar) im Miramichi River, New Brunswick, Canada (Lund et al. 2002). Stressproteine zeigten sich ebenso geeignet, zeitlich begrenzte Einleitungen in Gewässer zu detektieren. So wiesen Bachforellen (S. trutta f. fario) eines kleinen süddeutschen Gewässers (Katzenbach bei Rottenburg) an der Stelle vermuteter Einleitung kurz nach dem vermuteten Ereignis erhöhte Hsp70-Level in der Kieme und drei Wochen später in der Leber auf, was plausibel mit bekannten Aufnahme- und Speicherorten proteotoxischer Substanzen koinzidierte (Triebskorn et al. 2002). Über sog. ,Transplantationsexperimente', bei denen abundante Individuen innerhalb von Bachläufen versetzt werden, konnte in einer Studie mit Bachflohkrebsen (Gammarus fossarum) an der bereits erwähnten Körsch gezeigt werden, dass der Hsp70-Gehalt in diesem Fall weitgehend durch die aktuelle Situation im Habitat und nur zu einem weitaus geringeren Teil durch die Vorgeschichte der transplantierten Tiere bestimmt wurde (Schill und Köhler 2004a). Allerdings existieren aus terrestrischen Ökosystemen auch Gegenbeispiele, auf die im Folgenden eingegangen werden wird.

\section{Evolutionsbiologische Implikationen}

Es ist zum heutigen Kenntnisstand davon auszugehen, dass Populationen die Regulation ihrer Stressantwort den Verhältnissen in ihrem Habitat durch mikroevolutive Prozesse anpassen, vorausgesetzt, dass in diesen Habitaten ein gleichförmiger, über viele Generationen wirkender Selektionsdruck besteht. Insofern divergieren die Stressproteinantworten auf identische Stressoreinwirkung zwischen vorher unbelasteten Individuen und solchen, die Populationen aus entweder mit Chemikalien belasteten Habitaten (Eckwert und Köhler 1997; Knigge und Köhler 2000) oder klimatisch extremen Gebieten (z.B. dem Hochgebirge) entstammen (Sørensen et al. 2005). Obgleich die Resultate der meisten Arbeiten auf diesem Gebiet einander entsprechen, scheint die Richtung dieser evolutiven Anpassung nicht völlig einheitlich zu sein: Während bei Schnecken der Gattung Tegula 
thermotolerante Arten höhere Hsp70-Level zeigten (Tomanek 2005), wurde in einer Reihe von Studien nachgewiesen, dass bei über lange Zeit und viele Generationen exponierten Drosophila-Stämmen verminderte Hsp70-Expression auftrat (Bettencourt et al. 1999; Sørensen et al. 1999; Lansing et al. 2000) Das gleiche Phänomen wurde auch bei natürlichen Populationen von metallexponierten Collembolen, Asseln und Diplopoden (Köhler et al. 1999a; Köhler et al. 2000; Arts et al. 2004) und thermisch gestressten Drosophila (Sørensen et al. 2001; Zatsepina et al. 2001) gefunden. Auch gegenüber Cadmium vergleichsweise tolerante Stämme von Daphnia magna wurden nicht auf eine konstant hohe Hsp70-Produktion hin selektiert, sondern wiesen im Gegenteil nur eine sehr schwache Induzierbarkeit von Hsp70 durch Cadmium auf (Haap und Köhler, unpubliziert). Alle diese Studien legen nahe, dass ein experimentell nachgewiesener trade-off zwischen Hsp-Level und Reproduktionsrate (Krebs und Loeschcke 1994; Silbermann und Tatar 2000) wohl auch unter Freilandbedingungen existiert. Kristensen et al. (2008) wiesen nach, dass derartige Kosten insbesondere unter Freilandbedingungen in Defiziten, die unter Laborbedingungen nicht erkennbar waren, resultieren können. Vermutlich übersteigen die Kosten einer konstant hohen Hsp-Produktion deren protektive Vorteile, und deshalb ist anzunehmen, dass bei permanent exponierten Populationen ein Druck besteht, andere Wege der Toleranzentwicklung zu beschreiten. Die verminderte Expression von Hsp70, die nachweislich bei diesen Tieren nicht auf eine pathologische Schädigung metabolisch aktiver Gewebe zurückzuführen ist (Schill und Köhler 2004b) darf jedoch in keinem Fall dazu führen, die Exposition (d.h. die Intensität des Stressfaktors) zu unterschätzen: Da Stressproteine keine Marker der Exposition, sondern Effektmarker proteotoxischer Schädigung darstellen, darf auch hier nur eine Aussage zur Stresswirkung vorgenommen werden - und diese ist tatsächlich bei evolutiv angepassten Individuen deutlich geringer als bei sensitiven, Wildtyp'-Phänoptypen der gleichen Spezies.

Ein zentrales Dilemma der Evolutionsbiologie besteht darin, dass stabilisierende Selektion phänotypische Variation reduziert, wobei diese Variation jedoch die Grundvoraussetzung für den Angriff der Selektion darstellt. Somit ist davon auszugehen, dass Mechanismen existieren, die in der Lage sind, diesem Effekt entgegen zu arbeiten und phänotypische Variabilität zu erhalten. Bereits in den frühen 1940er-Jahren wurde ein unbekannter Mechanismus (später ,Waddington's widget' genannt) postuliert, der phänotypische Variabilität unterdrückt, so lange bis Organismen unter Stress geraten (Waddington 1942). In der Tat wurde erhöhte phänotypische Variation unter Stress oft beobachtet (z.B. Hoffmann und Parsons, 1997; Imasheva et al. 1997; Kristensen et al. 2003), die sich auch an die nächste Generation vererbte (Hoffmann und Merilä, 1999; Bubliy et al. 2000). Eine Reihe von Studien der letzten Jahre hat gezeigt, dass die
Proteinfamilie Hsp90 bei der Erklärung des Waddington's widget-Mechanismus eine zentrale Rolle spielen könnte (Ruden et al. 2003). So zeigten Rutherford und Lindquist (1998) bei Drosophila und Queitsch et al. (2002) bei Arabidopsis thaliana, dass Individuen aus Stämmen mit verminderter Hsp90-Expression zahlreiche morphologische Variationen in ihrer Individualentwicklung aufwiesen, die unter Stress besonders drastisch ausfielen, und schlossen hieraus, dass Hsp90 in der Lage ist, bei intakter Regulation diese morphologische Variation zu unterdrücken, d.h. als $\mathrm{Ca}$ pacitors der morphologischen Variabilität $\mathrm{zu}$ agieren, und die Individualentwicklung in einer Weise zu ,kanalisieren", dass Abweichungen von der ,normalen“ Entwicklung nicht zugelassen werden (Canalization). Spätere Studien wiesen nach, dass die Funktion von Hsp90 u. a. über epigenetische Mechanismen, d.h. über eine vererbbare Beeinflussung der Chromatinstruktur ausgeübt wird (Sollars et al. 2003; Ruden et al. 2005). Nach aktueller Ansicht existiert ,kryptische' Variabilität auf der Basis nicht realisierter Programme in wohl allen Populationen, und diese ist durch das Fehlen ihrer phänotypischen Realisation dem direkten Angriff der Selektion entzogen und kann somit aufrecht erhalten werden (Feder 2007). Obwohl das Capacitor-Konzept bisweilen kontrovers diskutiert wird, kann als evolutionsbiologischer ,Zweck' dieser ,gepufferten' kryptischen Variabilität die Möglichkeit angesehen werden, in dieser Weise mehrere zufällige, neutrale oder per se sogar nachteilige Mutationen akkumulieren zu können, um die Möglichkeit zur Evolvierbarkeit (evolvability) komplexer, letztlich vorteilhafter Adaptationen, welche mehr als eine einzige Mutation benötigen, aufrecht zu halten (Lauter und Doebley 2002; Feder 2007; Rutherford et al. 2007). Dies ist insbesondere bei einer Anpassung an sich rapide wandelnde Umweltbedingungen notwendig (Moczek 2007). Nach diesem Konzept würde die Canalization unter starkem Umweltstress ihre Funktion verlieren und die ,kryptische' (genetische) Variabilität eine reale phänotyische Variation hervorbringen. Es ist anzunehmen, dass auch die Stressanfälligkeit von CanalizationMechanismen unter Selektionsdruck steht, und in der Tat konnten mathematische Modelle zeigen, dass natürliche Selektion unter bestimmten Bedingungen die Evolution von solchen Canalization-Mechanismen favorisiert, die unter Extrembedingungen versagen (Eshel und Matessi 1998).

Nach neuesten Erkenntnissen existieren neben Hsp90 eine Reihe weiterer Capacitors, deren Effekte die Wirkung von Hsp90 auf die morphologische Variabilität komplementieren (Bergman und Siegal 2003; Suzuki und Nijhout 2006). So führten z.B. Mutationen in hormonellen Signaltransduktionskaskaden ebenfalls zu höherer morphologischer Variation (Suzuki und Nijhout 2006). Die Rolle der Stressproteinfamilie Hsp90 ist weitgehend unumstritten (Sangster und Queitsch, 2005; Rutherford et al. 2007), jedoch hat möglicherweise auch die Hsp70-Familie ähnliche Bedeutung, wie 
Abb. 3 Unterschiede in der Variation des Schalenmusters bei Populationen mediterraner Heideschnecken. Eine Population von Xeropicta derbentina (links) ohne nennenswerte Variation im Schalenmuster sowie eine stark polymorphe Population von Cernuella virgata (rechts)
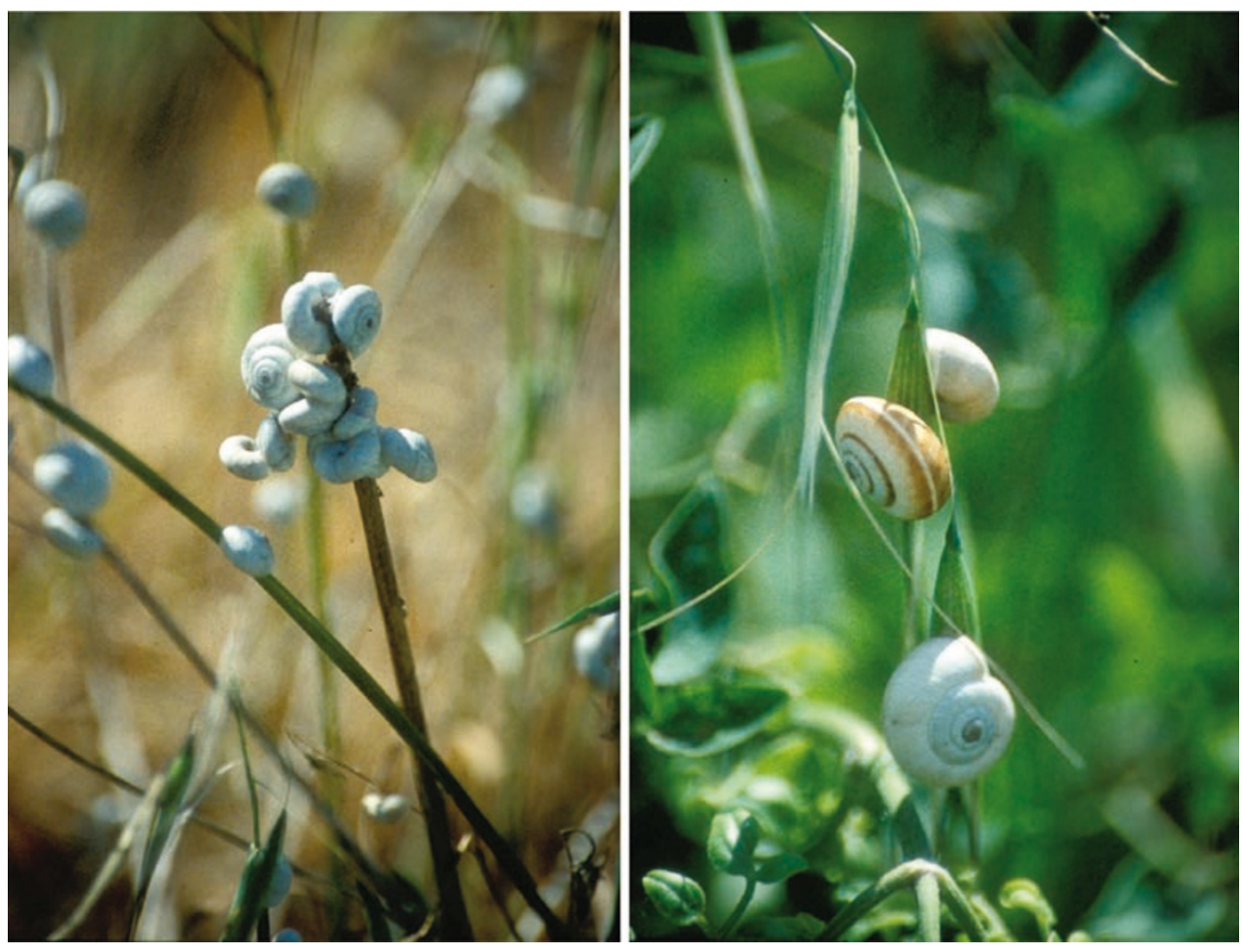

Ergebnisse bei Drosophila zum Auftreten morphologischer Missbildungen nahelegen (Roberts und Feder 1999). Trotz dieser in den letzten Jahren erzielten Ergebnisse ist das Potential von Capacitors bei Adaptationsprozessen gegenüber Umweltstressoren immer noch unbekannt, wie bereits vor einiger Zeit von Mitchell-Olds und Knight (2002) festgestellt wurde. Der Zusammenhang zwischen den Hsp90- und Hsp70-Leveln und phänotypischer Variation bei natürlichen Populationen in ihrem Lebensraum wurde bisher nur in einer Studie von Köhler et al. (2009) bzw. einer assoziierten Untersuchung an Cepaea hortensis (Lazzara, 2007) untersucht. Hier zeigte sich an bislang fünf Populationen terrestrischer Helicidae s. l. eine gute Korrelation zwischen der Höhe der maximalen Hsp70-Reaktion und der morphologischen Variabilität des Schalenmusters (Köhler et al. 2009, Abb. 3), was dafür spricht, dass Hsp70 eine Komponente von Waddington's widget auch unter natürlichen Einflüssen darstellen könnte. Für eine umfassende Aussage ist jedoch der Datensatz in den nächsten Jahren deutlich zu vergrößern. In jedem Fall jedoch spielen Stressproteine bei der physiologischen und evolutionsbiologischen Anpassung von Organismen an die in ihrem Habitat vorherrschenden Bedingungen eine zentrale Rolle.

\section{Literatur}

Arts M-JSJ, Schill RO, Knigge T, Eckwert H, Kammenga JE, Köhler H-R (2004) Stress proteins (hsp70, hsp60) induced in isopods and nematodes by field exposure to metals in a gradient near Avonmouth, UK. Ecotoxicology 13:739-755
Ashburner M, Bonner JJ (1979) The induction of gene activity in Drosophila by heat shock. Cell 17:241-254

Basu N, Todgham AE, Ackerman PA, Bibeau MR, Nakano K, Schulte PM, Iwama GK (2002) Heat shock protein genes and their functional significance in fish. Gene 295:173-183

Bettencourt BR, Feder FE, Cavicchi S (1999) Experimental evolution of Hsp70 expression and thermotolerance in Drosophila melanogaster. Evolution 53:484-492

Bergman A, Siegal ML (2003) Evolutionary capacitance as a general feature of complex gene networks. Nature 424:549-552

Bradley BP (1990) Stress proteins: their detection and uses in biomonitoring. ASTM (American Society for Testing and Materials) Special Technical Publication Suppl 1096:338-347

Bubliy OA, Loeschcke V, Imasheva AG (2000) Effect of stressful and nonstressful growth temperatures on variation of sternopleural bristle number in Drosophila melanogaster. Evolution 54:1444-1449

Cruz-Rodriguez LA, Chu, FLE (2002) Heat-shock protein (HSP70) response in the eastern oyster, Crassostrea virginica, exposed to PAHs sorbed to suspended artificial clay particles and to suspended field contaminated sediments. Aquat Toxicol 60:157-168

Dahlgaard J, Loeschcke V, Michalak P, Justesen J (1998) Induced thermotolerance and associated expression of the heat shock protein Hsp70 in adult Drosophila melanogaster. Funct Ecol 12:786-793

Eckwert H, Köhler H-R (1997) The indicative value of the hsp70 stress response as a marker for metal effects in Oniscus asellus (Isopoda) field populations: Variability between populations from metal-polluted and uncontaminated sites. Appl Soil Ecol 6:275-282

Eckwert H, Alberti G, Köhler H-R (1997) The induction of stress proteins (hsp) in Oniscus asellus (Isopoda) as a molecular marker of multiple heavy metal exposure. I. Principles and toxicological assessment. Ecotoxicology 6:249-262

Eder KJ, Köhler H-R, Werner I (2007) Pesticide and pathogen: Heat shock protein expression and acetylcholinesterase inhibition in juvenile Chinook salmon in response to multiple stressors. Environ Toxicol Chem 26:1233-1242 
Eder KJ, Leutenegger CM, Köhler H-R, Werner I (2009) Effects of neurotoxic insecticides on heat-shock proteins and cytokine transcription in Chinook salmon (Oncorhynchus tshawytscha). Ecotox Environ Saf 72:182-190

Eshel I, Matessi C, (1998) Canalization, genetic assimilation and preadaptation: a quantitative genetic model. Genetics 149:2119-2133

Fader SC, Yu Z, Spotila JR (1994) Seasonal variation in heat shock proteins (hsp70) in stream fish under natural conditions. J Thermal Biol 19:335-341

Feder ME (2007): Evolvability of physiological and biochemical traits: Evolutionary mechanisms including and beyond singlenucleotide mutation. J Exp Biol 210:1653-1660

Feder ME, Hofmann GE (1999) Heat-shock proteins, molecular chaperones, and the stress response: evolutionary and ecological physiology. Annu Rev Physiol 61:243-282

Ferguson IB, Snelgar W, Lay Yee M, Watkins CB, Bowen JH (1998) Expression of heat shock protein genes in apple fruit in the field. Aust J Plant Physiol 25:155-163

Gehring WJ, Wehner R (1995) Heat shock protein synthesis and thermotolerance in Cataglyphis, an ant from the Sahara desert. Proc Natl Acad Sci USA. 92:2994-2998

Gething M-J, Sambrook J (1992) Protein folding in the cell. Nature 355:33-45

Haap T, Triebskorn R, Köhler H-R (2008) Acute effects of diclofenac and DMSO to Daphnia magna: immobilisation and hsp70 induction. Chemosphere 73:353-359

Hallare AV, Köhler, H-R, Triebskorn R (2004) Developmental toxicity and stress protein responses in zebrafish embryos after exposure to diclofenac and its solvent, DMSO. Chemosphere 56:659-666

Hallare AV, Pagulayan R, Lacdan N, Köhler H-R, Triebskorn R (2005a): Assessing water quality in a tropical lake using biomarkers in zebtrafish embryos: developmental toxicity and stress protein responses. Environ Monit Assess 104:171-187

Hallare AV, Schirling M, Luckenbach T, Köhler H-R, Triebskorn R (2005b): Combined effects of temperature and cadmium on developmental parameters and biomarker responses in zebrafish (Danio rerio) embryos. J Therm Biol 30:7-17

Hallare AV, Nagel K, Köhler H-R, Triebskorn R (2006) Comparative embryotoxicity and proteotoxicity of three carrier solvents to zebrafish (Danio rerio) embryos. Ecotox Environ Saf 63:378-388

Hoffmann AA, Merilä J (1999) Heritable variation and evolution under favourable and unfavourable conditions. Trends Ecol Evol 14:96-101

Hoffmann AA, Parsons PA (1997) Consistent heritability changes under poor growth conditions. Trends Ecol Evol 12:460-461

Hofmann GE, Somero GN (1995) Evidence for protein damage at environmental temperatures: seasonal changes in levels of ubiquitin conjugates and hsp70 in the intertidal mussel Mytilus trossolus. J Exp Biol 198:1509-1518

Imasheva AG, Loeschcke V, Zhivotovsky LA, Lazebny OE (1997) Effect of extreme temperatures on phenotypic variation and developmental stability in Drosophila melanogaster and Drosophila buzzatii. Biol J Linnean Soc 61:117-126

Kammenga JE, Dallinger R, Donker MH, Köhler H-R, Simonsen V, Triebskorn R, Weeks JM (2000): Biomarkers in terrestrial invertebrates for ecotoxicological soil risk assessment. Rev Environ Contam Toxicol 164:93-147

Knigge T (2003) Subzelluläre Stressreaktionen von wirbellosen Bodenorganismen auf Metalle, Trockenheit und Temperatur. Dissertation, Universität Tübingen

Knigge T, Köhler H-R (2000) Lead impact on nutrition, energy reserves, respiration and stress protein (hsp70) level in Porcellio scaber (Isopoda) populations differently preconditioned in their habitats. Environ Pollut 108:209-217

Köhler H-R, Eckwert H (1997) The induction of stress proteins (hsp) in Oniscus asellus (Isopoda) as a molecular marker of multiple heavy metal exposure. II. Joint toxicity and transfer to field situations. Ecotoxicol 6:263-274

Köhler H-R, Triebskorn R, Stöcker W, Kloetzel P, Alberti G (1992) The $70 \mathrm{kD}$ heat shock protein (hsp70) in soil invertebrates: a possible tool for monitoring environmental toxicants. Arch Environ Contam Toxicol 22:334-338

Köhler H-R, Rahman B, Gräff S, Berkus M, Triebskorn R (1996) Expression of the stress-70 protein family (hsp 70) due to heavy metal contamination in the slug, Deroceras reticulatum: An approach to monitor sublethal stress conditions. Chemosphere 33:1327-1340

Köhler H-R, Belitz B, Eckwert H, Adam R, Rahman B, Trontelj P (1998) Validation of hsp70 stress gene expression as a marker of metal effects in Deroceras reticulatum (Pulmonata): Correlation with demographic parameters. Environ Toxicol Chem 17:2246-2253

Köhler H-R, Eckwert H, Triebskorn R, Bengtsson G (1999a) Interaction between tolerance and $70 \mathrm{kD}$ stress protein (hsp70) induction in collembolan populations exposed to long-term metal pollution. Appl Soil Ecol 11:43-52

Köhler H-R, Knödler C, Zanger M (1999b): Divergent kinetics of hsp70 induction in Oniscus asellus (Isopoda) in response to four environmentally relevant organic chemicals (B[a]P, PCB52, $\gamma$-HCH, PCP): Suitability and limits of a biomarker. Arch Environ Contam Toxicol 36:179-185

Köhler H-R, Zanger M, Eckwert H, Einfeldt I (2000) Selection favours low hsp70 levels in chronically metal-stressed soil arthopods. J Evol Biol 13:569-582

Köhler H-R, Bartussek C, Eckwert H, Farian K, Gränzer S, Knigge T, Kunz N (2001) The hepatic stress protein (hsp70) response to interacting abiotic parameters in fish exposed to various levels of pollution. J Aquat Ecosys Stress Recov 8:261-279

Köhler H-R, Alberti G, Seniczak S, Seniczak A (2005) Lead-induced hsp70 and hsp60 pattern transformation and leg malformation during post-embryonic development in the oribatid mite, Archegozetes longisetosus Aoki. Comp Biochem Physiol C 141:398-405

Köhler H-R, Sandu C, Scheil V, Nagy-Petrica EM, Segner H, Telcean I, Stan G, Triebskorn R (2007) Monitoring pollution in river Mureş, Romania, Part III: biochemical effect markers and integrative reflection. Environ Monit Ass 127:47-54

Köhler H-R, Lazzara R, Dittbrenner N, Capowiez Y, Mazzia C, Triebskorn R (2009) Snail phenotypic variation and stress proteins: do different heat response strategies contribute to Waddington's widget in field populations? J Exp Zool (Mol Dev Evol) 312B:136-147

Krebs RA, Loeschcke V (1994) Costs and benefits of activation of the heat shock response in Drosophila melanogaster. Funct Ecol 8:730-737

Kristensen TN, Dahlgaard J, Loeschcke V (2002) Inbreeding affects Hsp70 expression in two species of Drosophila even at benign temperatures. Evol Ecol Res 4:1209-1216

Kristensen TN, Dahlgaard J, Loeschcke V (2003) Effects of inbreeding and environmental stress on fitness - using Drosophila buzzatii as a model organism. Conservation Genet 4:453-465

Kristensen TN, Hoffmann AA, Overgaard J, Sørensen JG, Hallas R, Loeschcke V (2008) Costs and benefits of cold acclimation in field-released Drosophila. Proc Natl Acad Sci USA 105:216-221

Lansing E, Justesen J, Loeschcke V (2000) Variation in the expression of Hsp70, the major heat-shock protein, and thermotolerance in larval and adult selection lines of Drosophila melanogaster. J Thermal Biol 25:443-450

Lauter N, Doebley J (2002) Genetic variation for phenotypically invariant traits detected in teosinte: Implications for the evolution of novel forms. Genetics 160, 333-342

Lazzara R (2007) Mikroevolution morhologischer Variabilität, Thermotoleranz und die Induktion von Stressproteinen bei terrestrischen Pulmonaten. Diplomarbeit, Universität Tübingen, 111 S. 
Lund SG, Caissie D, Cunjak RA, Vijayan MM, Tufts BL (2002) The effects of environmental heat stress on heat-shock mRNA and protein expression in Miramichi Atlantic salmon (Salmo salar) parr. Can J Fish Aquat Sci 59:1553-1562

Minier C, Borghi V, Moore MN, Porte C (2000) Seasonal variation of MXD and stress proteins in the common mussel, Mytilus galloprovincialis. Aquat Toxicol 50:167-176

Mitchell-Olds T, Knight CA (2002) Evolution: chaperones as buffering agents? Science 296:2348-2349

Moczek AP (2007) Developmental capacitance, genetic accommodation, and adaptive evolution. Evol Dev 9:299-305

Morimoto RI, Jolly C, Satyal S, Mathew A, Shi Y, Kitagawa K (1999) Molecular chaperones and the heat shock response. Br J Cancer 80:S18

Mukhopadhyay I, Siddique HR, Bajpai VK, Saxena DK, Chowdhuri DK (2006) Syntheic pyrethroid cypermethrin induced cellular damage in reproductive tissues of Drosophila melanogaster: Hsp70 as a marker of cellular damage. Arch Environ Contamin Toxicol 51:673-680

Nazir A, Saxena DK, Chowdhuri DK (2003) Induction of hsp70 in transgenic Drosophila: biomarker of exposure against phthalimide group of chemicals. Biochim Biophys Acta - Gen Subj 1621:218-225

Nguyen HT, Joshi CP, Klueva N, Weng J, Hendershot KL, Blum A (1994) The reat-shock response and expression of heat-shock proteins in wheat under diurnal heat stress and field conditions. Aust J Plant Physiol 21:857-867

Nover L (1984) Heat shock response in eucaryotic cells. Springer, New York, $82 \mathrm{pp}$

Parsell DA, Lindquist S (1993) The function of heat-shock proteins in stress tolerance: degradation and reactivation of damaged proteins. Annu Rev Genet 27:437-496

Pockley AG (2003) Heat shock proteins as regulators of the immune response. Lancet 362:469-476

Podrabsky JE, Somero GN (2007) An inducible $70 \mathrm{kDa}$-class heat shock protein is constitutively expressed during early development and diapause in the annual killifish Austrofundulus limnaeus. Cell Stress and Chaperones 12:199-204

Queitsch C, Sangster TA, Lindquist S (2002) Hsp90 as a capacitor of phenotypic variation. Nature 417:618-624

Ritossa F (1962) A new puffing pattern induced by temperature shock and DNP in Drosophila. Experientia 18:571-573

Roberts SP, Feder ME (1999) Natural hyperthermia and expression of the heat shock protein Hsp70 affect developmental abnormalities in Drosophila melanogaster. Oecologia 121:323-329

Ruden DM, Garfinkel MD, Sollars VE, Lu X (2003) Waddington's widget: Hsp90 and the inheritance of acquired characters. Sem Cell Dev Biol 14:301-310

Ruden DM, Xiao L, Garfinkel MD, Lu X (2005) Hsp90 and environmental impacts on epigenetic states: A model for the trans-generational effects of diethylstilbestrol on uterine development and cancer. Human Mol Gen 14:R149-R155

Rutherford SL, Lindquist S (1998) Hsp90 as a capacitor for morphological evolution. Nature 396:336-342

Rutherford S, Hirate Y, Swalla BJ (2007) The Hsp90 capacitor, developmental remodelling, and evolution: The robustness of gene networks and the curious evolvability of metamorphosis. Crit Rev Biochem Mol Biol 42:355-372

Sanders BM (1993) Stress proteins in aquatic organisms: an environmental perspective. Crit Rev Toxicol 23:49-75

Sangster TA, Queitsch C (2005) The HSP90 chaperone complex, an emerging force in plant development and phenotypic plasticity. Curr Op Plant Biol 8:86-92

Saradha B, Vaithinathan S, Mathur PP (2008) Lindane alters the levels of HSP70 and clusterin in adult rat testis. Toxicology 243:116-123
Scheil, V, Triebskorn, R, Köhler, H-R (2008) Cellular and stress protein responses to the UV-filter 3-benzylidene camphor in the amphipod crustacean Gammarus fossarum (Koch 1835). Arch Environ Contam Toxicol 54:684-689

Scheil V, Kienle C, Osterauer R, Gerhardt A, Köhler H-R (im Druck) Effects of 3,4-dichloroaniline and diazinon on different biological organisation levels of zebrafish (Danio rerio) embryos and larvae. Ecotoxicology, DOI: 10.1007/s10646-008-0291-0

Schill RO, Köhler H-R (2004a) Does the environment or the source of the population define stress status and energy supply in the freshwater amphipod, Gammarus fossarum? Ecotoxicology 13:683-695

Schill RO, Köhler H-R (2004b) Energy reserves and metal-storage granules in the hepatopancreas of Oniscus asellus and Porcellio scaber (Isopoda) from a metal gradient at Avonmouth, UK. Ecotoxicology 13:787-796

Schill RO, Gayle PMH, Köhler H-R (2002) Daily stress protein (hsp70) cycle in chitons (Acanthopleura granulata Gmelin, 1791) which inhabit the rocky intertidal shoreline in a tropical ecosystem. Comp Biochem Physiol C 131:253-258

Schill RO, Görlitz H, Köhler H-R (2003) Laboratory simulation of a mining accident: acute toxicity, hsc/hsp70 response, and recovery from stress in Gammarus fossarum (Crustacea, Amphipoda) exposed to a pulse of cadmium. BioMetals 16:391-401

Schill RO, Steinbrück GHB, Köhler H-R (2004) Stress gene (hsp70) sequences and quantitative expression in Milnesium tardigradum (Tardigrada) during active and cryptobiotic stages. J Exp Biol 207:1605-1611

Schill RO, Pfannkuchen M, Fritz G, Köhler H-R, Brümmer F (2006) Quiescent gemmules of the freshwater sponge, Spongilla lacustris (Linnaeus, 1759), contain remarkably high levels of hsp70 stress protein and hsp70 stress gene mRNA. J Exp Zool 305A:449-457

Schill RO, Gayle PMH, Fritz GB, Köhler H-R (2008) Variability in the diurnal stress protein (Hsp70) cycle in tropical chitons (Acanthopleura granulata) from the intertidal zone: the influence of temperature and photoperiod exposure. Mar Freshw Behav Physiol 41:229-239

Schirling M, Triebskorn R, Köhler H-R (2004) Variation in stress protein levels (hsp70 and hsp90) in relation to oocyte development in Gammarus fossarum (Koch 1835). Inv Reprod Dev 45:161-167

Schirling M, Jungmann D, Ladewig V, Ludwichowski K-U, Nagel R, Köhler H-R, Triebskorn R (2006) Bisphenol A in artificial indoor streams: II. Stress response and gonad histology in Gammarus fossarum (Amphipoda). Ecotoxicology 15:143-156

Silbermann R, Tatar M (2000) Reproductive costs of heat shock protein in transgenic Drosophila melanogaster. Evolution 54:238-2045

Sollars V, Lu X, Xiao L, Wang X, Garfinkel MD, Ruden DM (2003) evidence for an epigenetic mechanism by which Hsp90 acts as a capacitor for morphological evolution. Nature Genetics 33:70-74

Sørensen JG, Loeschcke V (2001) Larval crowding in Drosophila melanogaster induces Hsp70 expression, and leads to increased adult longevity and adult thermal stress resistance. J Insect Physiol 47:1301-1307

Sørensen JG, Michalak P, Justesen J, Loeschcke V (1999) Expression of the heat-shock protein HSP70 in Drosophila buzzatii lines selected for thermal resistance. Hereditas 131:155-164

Sørensen JG, Dahlgaard J, Loeschcke V (2001) Genetic variation in thermal tolerance among natural populations of Drosophila buzzatii: down regulation of Hsp70 expression and variation in heat stress resistance traits. Funct Ecol 15:289-296

Sørensen JG, Kristensen TN, Loeschcke V (2003) The evolutionary and ecological role of heat shock proteins. Ecol Lett 6:10251037 
Sørensen JG, Norry FM, Scannapieco AC, Loeschcke V (2005) Altitudinal variation for stress resistance traits and thermal adaptation in adult Drosophila buzzatii from the New World. J Evol Biol 18:829-837

Sun W, van Montagu M, Verbruggen N (2002) Small heat shock proteins and stress tolerance in plants. Biochim Biophys Acta 1577:1-9

Sures B, Radszuweit H (2007) Pollution-induced heat shock protein expression in the amphipod Gammarus roeseli is affected by larvae of Polymorphus minutus (Acanthocephala). J Helminthol 81:191-197

Suzuki Y, Nijhout HF (2006) Evolution of a polyphenism by genetic accommodation. Science 311:650-652

Tissières A, Mitchell HK, Tracy UM (1974) Protein synthesis in the salivary glands of $D$. melanogaster. Relation to chromosome puffs. J Mol Biol 84:389-398

Tomanek L (2005) Two-dimensional gel analysis of the heat-shock response in marine snails (genus Tegula): Interspecific variation in protein expression and acclimation ability. J Exp Biol 208: 3133-3143

Triebskorn R, Böhmer J, Braunbeck T, Honnen W, Köhler H-R, Lehmann R, Oberemm A, Schwaiger J, Segner H, Schüürmann G, Traunspurger W (2001) The project VALIMAR (VALIdation of bioMARkers for the assessment of small stream pollution): objectives, experimental design, summary of results, and recommendations for the application of biomarkers in risk assessment. J Aquat Ecosys Stress Recov 8:161-178

Triebskorn R, Adam S, Casper H, Honnen W, Pawert M, Schramm M, Schwaiger J, Köhler H-R (2002) Biomarkers as diagnostic tools for evaluating effects of unknown past water quality conditions on stream organisms. Ecotoxicology 11:451-465

Ulmasov HA, Karaev KK, Lyashko VN, Evgenev MB (1993) Heat shock response in camel (Camelus dromedarius) blood cells and adaptation to hyperthermia. Comp Biochem Physiol B 106:867-872

Waddington C (1942) Canalization of development and the inheritance of acquired characters. Nature 150:563-565

Weber LP, Janz DM (2001) Effect of beta-naphthoflavone and dimethylbenz[a]anthracene on apoptosis and HSP70 expression in juvenile channel catfish (Ictalurus punctatus) ovary. Aquat Toxicol 54:39-50
Weber LP, Diamond SL, Bandiera SM, Janz DM (2002) Expression of HSP70 and CYP1A protein in ovary and liver of juvenile rainbow trout exposed to beta-naphthoflavone. Comp Biochem Physiol C $131: 387-394$

Werner I (2004) The influence of salinity on the heat-shock protein response of Potamocorbula amurensis (Bivalvia). Mar Environ Res 58:803-807

Werner I, Hinton DE (1999) Field validation of hsp70 stress proteins as biomarkers in Asian clam (Potamocorbula amurensis): is downregulation an indicator of stress? Biomarkers 4:473-484

Werner I, Hinton DE (2000) Spatial profiles of hsp70 proteins in Asian clam (Potamocorbula amurensis) in northern San Francisco Bay may be linked to natural rather than anthropogenic stressors. Mar Environ Res 50:379-384

Werner I, Viant MR, Rosenblum ES, Gantner AS, Tjeerdema RS, Johnson ML (2006) Cellular responses to temperature stress in steelhead trout (Oncorhynchus mykiss) parr with different rearing histories. Fish Physiol Biochem 32:261-273

Yeh FL, Hsu T (2002) Differential regulation of spontaneous and heatinduced HSP 70 expression in developing zebrafish (Danio rerio). J Exp Zool 293:349-359

Yost HJ, Lindquist S (1986) RNA splicing is interrupted by heat shock and is rescued by heat shock protein synthesis Cell 45:185-193

Yost HJ, Lindquist S (1991) Heat shock proteins affect RNA processing during the heat shock response of Saccharomyces cerevisiae. Mol Cell Biol 11:1062-1068

Zanger M, Köhler H-R (1996) Colour change: A novel biomarker indicating sublethal stress conditions in the millipede Julus scandinavius (Diplopoda). Biomarkers 1:99-106

Zanger M, Alberti G, Kuhn M, Köhler H-R (1996) The stress-70 (hsp70) protein family in diplopods: induction and characterization. J Comp Physiol B 165:622-627

Zatsepina OG, Velikodvorskaia VV, Molodtsov VB, Garbuz D, Lerman DN, Bettencourt BR, Feder ME, Evgenev MB (2001) A Drosophila melanogaster strain from sub-equatorial Africa has exceptional thermotolerance but decreased Hsp70 expression. J Exp Biol 204:1869-1881

Zheng S, Sun T, Xiao H, Qiu X, Song Y (2008) Low dose benzo(a) pyrene up-regulated the transcription of HSP70 and HSP90 in Eisenia fetida. Yingyong Shengtai Xuebao 19:401-406 